\title{
Práctica de mastografías y pruebas de Papanicolaou entre mujeres de áreas rurales de México
}

\author{
Sandra G Sosa-Rubí, D en Econ, (') Dilys Walker, MD, PhD, (') Edson Serván, M en Econ.(')
}

\section{Sosa-Rubí SG,Walker D, Serván E. Práctica de mastografías y pruebas de Papanicolaou entre mujeres de áreas rurales de México. Salud Publica Mex 2009;5 I supl 2:S236-S245.}

\section{Resumen}

Objetivo.Analizar la práctica de mastografías y Papanicolaou y el seguimiento de resultados anormales entre mujeres de áreas rurales en México. Material y métodos. Se realizó un estudio de corte transversal con la Encuesta de Evaluación de los Hogares Rurales (ENCEL 2007)- Oportunidades levantada entre julio y noviembre del año 2007. Se utilizó un modelo logístico multinivel para modelar la realización de mastografías y Papanicolaou. Resultados. Se encontró una baja frecuencia en la ejecución de mastografías en mujeres de 30 a 39 años (12\%) y de 40 a 49 años (16\%), además de un bajo informe de seguimiento médico $(60 \%)$ en los grupos de edad de alto riesgo y con resultados anormales. Estos factores se relacionaron con la falta de acceso a recursos médicos y la condición indígena de las mujeres. Conclusión. Se recomienda fortalecer el acceso a exámenes de mastografía y Papanicolaou entre mujeres de alto riesgo en áreas rurales y el seguimiento adecuado de casos anormales para reducir la letalidad del cáncer de mama (CaMa) y el cáncer cervicouterino $(\mathrm{CaCu})$.

Palabras clave: cáncer de mama; cáncer cervicouterino; mastografía; Papanicolaou; zonas rurales; México
Sosa-Rubí SG, Walker D, Serván E.

Performance of mammography and Papanicolau among rural women in Mexico.

Salud Publica Mex 2009;5 I suppl 2:S236-S245.

\section{Abstract}

Objective. To analyze the frequency of reporting a recently conducted mammogram and/or pap smear and follow-up of abnormal findings among rural poor women in Mexico. Methods. We performed a cross-sectional analysis using data collected in the ENCEL 2007 - Oportunidades survey carried out between July - November, 2007. We used multilevel logistic regression to model the use of mammography and pap smears. Results. We found a low frequency of reported recent mammogram among the rural poor from 30-39 years old (12\%) and $40-49$ years old (16\%) and a low frequency of reported medical follow-up of abnormal findings (60\%), particularly among women at higher risk because of age or abnormal findings. These findings were associated with a lack of availability of medical resources and being of indigenous origin. Conclusion. It is fundamental to strengthen both access to and follow-up of mammography and papsmears, particularly among high-risk rural women in order to reduce the level of mortality associated with breast and cervicaluterine cancer.

Key words: breast cancer; cervical-uterine cancer; mammography; Pap; rural areas; Mexico

(I) Instituto Nacional de Salud Pública. Cuernavaca, México.

Fecha de recibido: 3 de noviembre de 2008 - Fecha de aprobado: 8 de enero de 2009

Solicitud de sobretiros: Dra. Sandra G. Sosa-Rubí. Instituto Nacional de Salud Pública. Av. Universidad 655, col. Santa María Ahuacatitlán. 62508 Cuernavaca, Morelos.

Correo electrónico: srubi@correo.insp.mx 
E número de fallecimientos por cáncer de mama CaMa) se ha duplicado en poco más de 20 años y afecta tanto a países industrializados como a los menos desarrollados., ${ }^{1,2}$ En la actualidad, el cáncer de mama representa un problema de salud pública en México. ${ }^{3}$ Al igual que los estándares mundiales y de América Latina, es la segunda causa de muerte por afección maligna en mujeres mayores de 25 años. En 2006, la tasa de mortalidad por cáncer de mama era de 15.8 por cada 100000 habitantes. $^{4}$

Aunque los factores de riesgo relacionados con esta malformación no se han definido 5 aún con claridad, su detección en estadios tempranos reduce sus niveles deletalidad. En consecuencia, resulta clave el acceso oportuno a los servicios de salud preventivos para el control exitoso del CaMa. No obstante, en México la mayor parte de los casos (casi 90\%) se diagnostica en etapas tardías. ${ }^{6}$

En 2000 se notificaron 471000 nuevos casos de cáncer cervicouterino en el mundo, de los cuales se informaron 288000 muertes. Alrededor de $80 \%$ de esas muertes ocurrió en países en vías de desarrollo. ${ }^{7}$ Aunque la tasa de mortalidad por $\mathrm{CaCu}$ ha disminuido, aún representa la primera causa de muerte por neoplasias en mujeres mayores de 25 años en México. ${ }^{4}$ En el año 2006, la tasa de mortalidad por $\mathrm{CaCu}$ fue de 16.8 por cada 100000 mujeres. ${ }^{4}$ El peso del CaCu en países como México se ha vinculado con la falta de un programa eficaz para el pronóstico efectivo de esta tumoración. ${ }^{8}$ La conformación de un programa de detección con coberturas elevadas en mujeres de 25 a 65 años, la adecuada toma e interpretación de la muestra, y el diagnóstico y tratamiento oportunos son factores que pueden contribuir a la efectividad del programa. ${ }^{8,9}$ Sin embargo, se ha encontrado que las mujeres que viven en zonas rurales tienen un inadecuado acceso al examen del Papanicolaou en México. ${ }^{10}$

Tanto para el CaMa como para el CaCu, la detección oportuna es clave para reducir la mortalidad por ambos padecimientos. El acceso regular a los servicios de salud calificados para la detección oportuna y el seguimiento temprano y apropiado de resultados anormales en ambos tipos de malformaciones contribuyen de manera nodal a la reducción de dichas tasas. El propósito de este artículo es analizar la realización de pruebas de prevención para CaMa y CaCu y el seguimiento médico de casos anormales en mujeres de zonas rurales que viven en localidades cubiertas por el programa Oportunidades. Se analiza la práctica de pruebas de mastografías y Papanicolaou en los últimos 12 meses en mujeres de 20 a 49 años y mastrografía y Papanicolaou en los últimos tres años en mujeres de 65 años y mayores.

En áreas rurales, la frecuencia con la que se informa la práctica de pruebas de Papanicolaou es alta, dado el fortalecimiento de los programas de detección oportuna del $\mathrm{CaCu}$ en los últimos 20 años en el plano nacional. ${ }^{11}$ Sin embargo, se asume que dicho acceso es todavía diferenciado entre mujeres ubicadas en localidades con mayor disponibilidad de recursos de salud y otras pacientes. Se presupone que la frecuencia en la realización de mastografías en áreas rurales debe ser considerablemente más limitada debido el requerimiento de capacidad de infraestructura específica para la práctica de éstas.

\section{Material y métodos}

Se llevó a cabo un estudio de corte transversal que usa información sobre la realización de exámenes de detección de cáncer de mama y cáncer cervicouterino de la Encuesta de Evaluación de los Hogares Rurales (ENCEL 2007) efectuada entre julio y noviembre del año 2007. ${ }^{12}$ La ENCEL 2007 es representativa de localidades rurales que cumplen con los requisitos para ser incorporadas al programa Oportunidades* en 13 estados de la República. ${ }^{\ddagger}$ Esta encuesta se compone de 868 localidades con 43887 hogares. Se seleccionó una submuestra de 13614 mujeres con información sobre salud reproductiva preventiva. $\S$ Después de eliminar variables de resultado y algunas covariables ${ }^{\#}$ con valores perdidos, se obtuvo una muestra de 7428 mujeres de 20 a 49 años de edad. Lo mismo se hizo con mujeres de 65 años y mayores, con las cuales se conformó una muestra de 1325 personas.

A las mujeres de 20 a 49 años se les preguntó sobre la realización de pruebas de Papanicolaou y mastrografía en los últimos 12 meses, mientras que a las de 65 y más años se las cuestionó acerca de la práctica de pruebas de Papanicolaou y mastografía en los últimos tres años. ${ }^{\infty}$

\footnotetext{
* La identificación de hogares como beneficiarios de Oportunidades se realizó en tres etapas. Primero se seleccionaron las localidades con mayor índice de marginación con base en datos censales. Segundo, dentro de las localidades seleccionadas, se identificaron los hogares en pobreza extrema mediante la información de una encuesta socioeconómica recabada en cada hogar. La selección de los hogares beneficiarios de Oportunidades se realizó a través de la aproximación inicial a la condición de pobreza, con la utilización de una línea de pobreza. Tercero, la lista de los beneficiarios potenciales se presenta ante asambleas comunitarias para su revisión y discusión. ${ }^{13}$

₹ Aguascalientes, Chiapas, Durango, Guerrero, Hidalgo, Michoacán, Nayarit, Oaxaca, Puebla, Querétaro, San Luis Potosí, Sinaloa y Veracruz.

§ Esta submuestra se compone de mujeres jefas del hogar de 20 a 49 años y mujeres adultas de 65 años y mayores.

\# Se eliminaron 2792 valores perdidos en las variables de resultado y 2032 valores perdidos de algunas covariables.

${ }^{\infty}$ Es importante señalar que dado que las mujeres de 65 años y mayores deben recordar la relación de alguna de las pruebas en un lapso de tiempo mayor es posible que se presente un sesgo de memoria.
} 
En ambos grupos se investigó sobre su acceso a los resultados de las pruebas de Papanicolaou y mastografía; el seguimiento médico en caso de resultados anormales; y el lugar del seguimiento médico. ${ }^{*}$

\section{Variables}

En el análisis multivariado se emplearon dos variables de resultado: la primera es una variable dicotómica que indica si la mujer se sometió a una prueba de Papanicolaou en los últimos 12 meses y la segunda revela si la mujer fue objeto de una mastografía en los últimos 12 meses. Para el análisis de correlación simple se analizó la realización de mastografías y Papanicolaou entre mujeres de 65 años y mayores.

Se incluyeron indicadores de exposición al Programa Oportunidades: el primero es el autorreporte de la mujer sobre su pertenencia a Oportunidades; el segundo es el periodo de incorporación de la localidad al programa (1998, 1999-2000, 2001-2003 y 2004-2007). Con esta variable se asume que existe un efecto diferenciado sobre el acceso a consultas reproductivas preventivas en mujeres que viven en localidades con mayor antigüedad en el programa respecto de aquéllas en localidades con menor tiempo. Se presupone que las mujeres pertenecientes a localidades con mayor duración en el programa podrían tener más disposición a utilizar otro tipo de servicios preventivos de salud como parte del efecto aprendizaje derivado de su exposición a los programas de prevención promovidos por Oportunidades.

Como covariables se incluyeron características individuales de la mujer: edad, estado marital, trabajo en la última semana, paridad de al menos un hijo y educación. A nivel del hogar se consideraron la condición indígena, variables que indican la condición de aseguramiento (IMSS, ISSSTE, IMSS-Oportunidades, Seguro Popular) y el índice de activos como aproximación al nivel socioeconómico del hogar. Se incluyeron además variables que indican la disponibilidad de médicos y enfermeras a nivel municipal, lo que permite tener una idea del acceso general a recursos médicos en áreas rurales con más/menos disponibilidad de recursos médicos, y que en algunos casos podría ser sinónimo del acceso a más / menos tecnología médica. ${ }^{\ddagger}$ Aunque lo ideal sería incluir información específica sobre el acceso

\footnotetext{
* No se consideró a mujeres de 50 a 64 años porque no se contó con información sobre salud preventiva de estas personas en la encuesta analizada.

₹ En realidad, se asume que la mayor concentración de recursos médicos a nivel municipal puede correlacionarse con la disponibilidad de tecnología.
}

a patólogos y equipos de rayos $\mathrm{X}$ para la realización de mastrografías a nivel municipal, no se dispone de esa información específica.

\section{Métodos estadísticos}

En el análisis multivariado se utilizó un modelo logístico multinivel con el objetivo de relajar el supuesto de independencia de las respuestas de las mujeres pertenecientes a la misma localidad. Se asume que la actitud de las mujeres de una misma localidad hacia el uso de servicios de salud preventivo puede correlacionarse debido a que están expuestas a los mismos programas preventivos de salud. Este modelo permite incluir un intercepto aleatorio específico por cada localidad $\xi \sim \mathrm{N}(0, \psi)$ en el predictor lineal. Se obtiene un modelo logístico con un resultado dicotómico $\mathrm{y}_{i j}$ para cada mujer $i$ ubicada en una localidad $j$, donde $y_{i j}=1$ si la mujer se sometió a un examen de Papanicolaou en los últimos 12 meses e igual a cero en caso contrario. La misma lógica se sigue con el análisis empírico de la realización de mastografía, $\mathrm{y}_{i j}=1$ si la mujer fue objeto de una mastografía en los últimos 12 meses. El modelo se describe como:

$\operatorname{logit}\left\{\operatorname{Pr}\left(\mathrm{y}_{i j}=1 \mid \mathrm{x}_{2 j^{j}} \mathrm{x}_{3 j \ldots} \mathrm{x}_{n j^{\prime}} \zeta_{j j}\right)\right\}=\beta_{1}+\beta_{2} \mathrm{x}_{2 j}+\beta_{3} \mathrm{x}_{3 i j}+\beta_{4} \mathrm{x}_{4 j}+\ldots+\beta_{n} \mathrm{x}_{n j}+\zeta j$

entonces $\mathrm{y}_{i j}$ depende de un grupo de variables de $\mathrm{x}_{2 j}$ a $\mathrm{x}_{n j}$ que miden los efectos fijos $\mathrm{y}$ un intercepto aleatorio $\zeta_{j}$ que cuantifica los efectos aleatorios. El modelo se estimó mediante el comando gllamm disponible en el programa StataCorp 9.2. ${ }^{14}$ Para la muestra de mujeres de 65 y más años se realizó un análisis descriptivo con correlaciones simples.

\section{Resultados}

En el cuadro I se muestran las estadísticas descriptivas sobre el acceso a pruebas de Papanicolaou y mastografía de mujeres de $20^{*}$ a 49 años y de 65 y más años. El cuadro muestra la media y la desviación estándar de las covariables. Hasta $65 \%$ de las mujeres de 20 a 49 años se sometió a una prueba de Papanicolaou en los últimos 12 meses. Al analizar los grupos de edad se advierte que el porcentaje de mujeres que fue objeto de pruebas de Papanicolaou aumenta con la edad: en $53 \%$ de las mujeres de 20 a 29 años se efectuó la prueba de Papani-

\footnotetext{
₹ De acuerdo con The American College of Obstetrician and Gynecologist, el primer examen de Papanicolaou en mujeres jóvenes se recomienda no más tarde de los 21 años $^{15}$ y por ello el punto de corte es de 20 años de edad.
} 


\section{Cuadro I}

Utilización de los servicios preventivos de salud reproductiva: pruebas de Papanicolaou y mastografía. MÉXICO, OCTUBRE 2008

\begin{tabular}{|c|c|c|c|c|c|c|c|}
\hline \multicolumn{4}{|c|}{ Realización de pruebas de Papanicolaou y seguimiento } & \multicolumn{4}{|l|}{ Realización de mastografia y seguimiento } \\
\hline & $N$ & Media & $D E$ & & N & Media & $D E$ \\
\hline Realización del Papanicolaou en los últimos 12 meses & & & & Realización de la mastografía en los últimos 12 meses & & & \\
\hline Mujeres de 20 a 49 años de edad & 9460 & 0.65 & 0.48 & Mujeres de 20 a 49 años de edad & 9513 & 0.12 & 0.32 \\
\hline Por grupos de edad & & & & Por grupos de edad & & & \\
\hline Mujeres de 20 a 29 años de edad & 2678 & 0.53 & 0.50 & Mujeres de 20 a 29 años de edad & 2687 & 0.08 & 0.27 \\
\hline Mujeres de 30 a 39 años de edad & 3873 & 0.68 & 0.47 & Mujeres de 30 a 39 años de edad & 3897 & 0.12 & 0.32 \\
\hline Mujeres de 40 a 49 años de edad & 2909 & 0.71 & 0.45 & Mujeres de 40 a 49 años de edad & 2929 & 0.16 & 0.37 \\
\hline Recibieron resultados & & & & Recibieron resultados & & & \\
\hline Mujeres de 20 a 49 años de edad & 6099 & 0.72 & 0.45 & Mujeres de 20 a 49 años de edad & $|13|$ & 0.80 & 0.40 \\
\hline Resultados normales & & & & Resultados normales & & & \\
\hline Mujeres de 20 a 49 años de edad & 4387 & 0.96 & 0.20 & Mujeres de 20 a 49 años de edad & 896 & 0.94 & 0.23 \\
\hline Seguimiento médico de casos con resultado anormal & & & & Seguimiento médico de casos con resultado anormal & & & \\
\hline Mujeres de 20 a 49 años de edad & 185 & 0.86 & 0.35 & Mujeres de 20 a 49 años de edad & 49 & 0.71 & 0.46 \\
\hline Realización del Papanicolaou en los últimos tres años & & & & Realización de la mastografía en los últimos tres años & & & \\
\hline Mujeres de 65 y más años & 1368 & 0.87 & 0.34 & Mujeres de 65 y más años & 2337 & 0.07 & 0.26 \\
\hline Resultados normales & & & & Resultados normales & & & \\
\hline Mujeres de 65 y más años & 944 & 0.04 & 0.19 & Mujeres de 65 y más años & 135 & 0.96 & 0.21 \\
\hline Seguimiento médico de casos con resultado anormal & & & & Seguimiento médico de casos con resultado anormal & & & \\
\hline Mujeres de 65 y más años & & & & Mujeres de 65 y más años & 6 & 0.33 & 0.52 \\
\hline
\end{tabular}

colaou en los últimos 12 meses, en $68 \%$ de las mujeres en el grupo de edad de 30 a 39 años y en $71 \%$ del grupo de edad de 40 a 49 años. En personas mayores de 65 años se encontró que $87 \%$ se sometió a una prueba de Papanicolaou en los últimos tres años, una proporción que no cambia cuando se comparan subgrupos de edad entre estas mujeres. ${ }^{*}$ Entre mujeres de 20 a 49 años que fueron objeto de Papanicolaou, $70 \%$ recibió sus resultados y éstos fueron normales en una proporción de 96\%; este porcentaje desciende en un punto porcentual

\footnotetext{
* Este resultado debe considerar la posible presencia de sesgo de memoria por el lapso de tiempo (tres años) en el que las mujeres deben recordar la realización del Papanicolaou o mastografía.
}

en el grupo de edad de 40 a 49 años. De las mujeres con resultados anormales, $86 \%$ tuvo seguimiento médico y de esa cifra $40 \%$ recibió dicho seguimiento médico en la Secretaría de Salud.

En contraste, sólo $12 \%$ de las mujeres de 20 a 49 años se sometió a una mastografía en los últimos 12 meses. Cuando se analiza por grupos de edad, esta proporción no aumenta en grado considerable en los grupos de mujeres con mayor edad: $8 \%$ de mujeres de 20 a 29 años fue objeto de una mastografía en los últimos 12 meses, $12 \%$ en pacientes de 30 a 39 años y $16 \%$ en mujeres de 40 a 49 años. En personas mayores de 65 años se encontró que 7\% se sometió a una mastografía en los últimos tres años, una proporción que no cambia si se comparan los grupos de edad de las mujeres mayores de 65 años. En el grupo de 20 a 49 años, 80\% 
recibió los resultados de la mastografía y éstos fueron normales en una proporción de $94 \%$. Este porcentaje decrece en un punto porcentual para el grupo de edad de 40 a 49 años. En $71 \%$ de los casos anormales del grupo de edad de 20 a 49 años, las mujeres tuvieron seguimiento médico. Sin embargo, al analizar este dato por grupos de edad se observa que apenas $60 \%$ de las pacientes con resultados anormales en mujeres de 40 a 49 años tuvo seguimiento médico y en la mayoría de los casos éste lo suministraron el IMSS o la Secretaría de Salud. Cuando se analizó el seguimiento de los resultados anormales por población con distintos beneficios se encontró que más de $80 \%$ de las personas con resultados anormales en el Papanicolaou, ubicadas en cualquiera de los grupos con beneficios, es decir, con Oportunidades, IMSS / ISSSTE, Seguro Popular o uso de IMSS-Oportunidades, tiene seguimiento con un médico de estos resultados. En el caso de los resultados de la mastografía, las proporciones descienden en aquellas que tienen IMSS-Oportunidades $(60 \%)$ y Seguro Popu$\operatorname{lar}(74 \%){ }^{*}$

\section{Resultados del modelo multivariado para mujeres de 20 a 49 años}

El cuadro II muestra la descripción de las principales covariables utilizadas para el ajuste del modelo logístico multinivel. La edad promedio de las mujeres de esta muestra es de 35 años; ${ }^{\neq}$hasta $93 \%$ de estas mujeres tiene una relación conyugal y $84 \%$ posee al menos un hijo. La mayor parte es alfabeta $(84 \%)$ y tiene en promedio seis años de educación. Una baja proporción de estas mujeres (19\%) trabaja en algún empleo formal o informal; la proporción restante corresponde a amas de casa. El 22\% tiene origen indígena y $66 \%$ menciona que su familia es beneficiaria del programa Oportunidades. Hasta $6 \%$ posee algún tipo de aseguramiento (IMSS, ISSSTE) y 27\% está afiliado al Seguro Popular. El índice de activos promedio (los activos acumulados en el hogar) $)^{\S}$ es bajo si se consideran los valores mínimo (-1.31)

\footnotetext{
* Los resultados no se muestran en el artículo.

* Es necesario considerar que la muestra analizada es representativa de las jefas de hogar y que para el análisis multivariado se consideró sólo a las mujeres de 20 a 49 años.

$\S$ Para establecer un criterio de estratificación de los hogares se definió un índice de riqueza como principal indicador de nivel socioeconómico. Dicho índice se construyó a través de la matriz de correlación policórica de un análisis de componentes principales. ${ }^{16}$ Se consideraron variables de posesión de activos en el hogar, como estufa, refrigerador, televisores, teléfono, etcétera.
}

y máximo (4.37); este resultado no es extraordinario si se considera que se trata de mujeres que viven en zonas rurales pobres.

En la localidad se encontró que $18 \%$ de estas mujeres vive en sitios con un índice de marginación alto, $39 \%$ en puntos con un índice de marginación medio y $43 \%$ en lugares con un índice de marginación bajo. Una proporción de $64 \%$ de estas localidades cuenta con al menos una unidad de salud disponible, lo cual supone que en las localidades restantes (36\%) las mujeres deben desplazarse a otras localidades para tener acceso a servicios preventivos. En el plano municipal se cuenta en promedio con seis médicos por cada 1000 habitantes y cuatro centros de salud por cada 1000 habitantes. ${ }^{*}$

En el cuadro III se muestran los resultados del modelo logístico multinivel. En la primera columna de este cuadro se exponen los resultados para la realización de alguna prueba de Papanicolaou en los últimos 12 meses. Se halló que pertenecer al grupo de edad de 40 a 49 años se relaciona de manera significativa con el hecho de someterse a una prueba de Papanicolaou en los últimos 12 meses $(R M=1.49)$. La misma relación significativa se encontró entre mujeres casadas $(R M=$ 1.44) y que trabajan $(R M=1.19)$. Se observó un nexo significativo entre pertenecer a Oportunidades y ser objeto de una prueba en los últimos 12 meses $(R M=3.23)$. De manera adicional, se encontró que las mujeres que pertenecen a localidades con mayor tiempo en el programa, en particular aquéllas beneficiadas desde 1998, tienen una vinculación significativa con la realización de pruebas $(R M=1.40)$.

El aseguramiento $(R M=1.27)$ o la afiliación al Seguro Popular $(R M=1.38)$ tienen una relación significativa con la práctica de exámenes de Papanicolaou; en cambio, la condición indígena de las mujeres se vincula en grado notable con la falta de realización de la prueba de Papanicolaou $(R M=0.76)$. La disponibilidad de algún centro de salud en la localidad $(R M=1.22)$ y la mayor proporción de médicos a nivel municipal $(R M=1.18)$ contribuyen de forma notoria a la práctica de pruebas de Papanicolaou. Por último, se halló que las mujeres que residen en localidades con un índice de marginación

\footnotetext{
* Este promedio asume que existe una distribución homogénea de recursos entre las localidades que componen el municipio, cuando en realidad es muy probable que haya localidades que no cuenten con recursos médicos (médicos y centros de salud). Al tomar en cuenta la falta de información sobre la disponibilidad de patólogos o equipos a nivel municipal, esta medida es la que mejor aproxima el acceso promedio a recursos de salud.
} 
Cuadro II

Estadísticas descriptivas: muestra de mujeres de 20 a 49 años. México, octubre 2008

\begin{tabular}{|c|c|c|c|c|c|}
\hline Características & $N$ & Media & $D E$ & Min & Max \\
\hline Individuales: & 9460 & 34.88 & 7.68 & 20 & 49 \\
\hline Edad en años & 9460 & 0.28 & 0.45 & 0 & 1 \\
\hline Mujer de 20 a 29 años de edad & 9460 & 0.41 & 0.49 & 0 & 1 \\
\hline Mujer de 30 a 39 años de edad & 9460 & 0.31 & 0.46 & 0 & 1 \\
\hline Mujer de 40 a 49 años de edad & 9456 & 0.93 & 0.26 & 0 & 1 \\
\hline Casada & 9226 & 0.84 & 0.36 & 0 & 1 \\
\hline Tiene al menos un hijo & 9460 & 0.19 & 0.40 & 0 & 1 \\
\hline Trabaja & 8582 & 0.84 & 0.37 & 0 & 1 \\
\hline Alfabeta & 7428 & 5.61 & 2.78 & 0 & 18 \\
\hline Años de escolaridad & 7428 & 0.77 & 0.42 & 0 & 1 \\
\hline Primaria & 7428 & 0.19 & 0.40 & 0 & 1 \\
\hline Secundaria & 7428 & 0.04 & 0.19 & 0 & 1 \\
\hline
\end{tabular}

Superior

\begin{tabular}{llllll} 
Del hogar: & 9162 & 0.66 & 0.47 & 0 & I \\
\hline Incorporado a Oportunidades (autorreporte) & 9427 & 0.22 & 0.41 & 0 & I \\
\hline Condición indígena del jefe del hogar o esposa & & & & & \\
\hline Condición de aseguramiento: & 9460 & 0.06 & 0.23 & 0 & I \\
\hline IMSS o ISSSTE & 9460 & 0.15 & 0.36 & 0 & I \\
\hline IMSS-Oportunidades & 9460 & 0.27 & 0.45 & 0 & I \\
\hline Seguro Popular & 9460 & 0.22 & 0.92 & -1.31 & 4.37 \\
\hline
\end{tabular}

Índice de activos (aprox. de nivel socioeconómico)

\begin{tabular}{|c|c|c|c|c|c|}
\hline De la localidad: & 9420 & 0.36 & 0.48 & 0 & I \\
\hline Grado de marginación bajo & 9420 & 0.32 & 0.47 & 0 & I \\
\hline Grado de marginación medio & 9420 & 0.32 & 0.46 & 0 & 1 \\
\hline Grado de marginación alto & 8813 & 0.64 & 0.48 & 0 & I \\
\hline \multicolumn{6}{|l|}{ Disponibilidad de un centro de salud } \\
\hline Periodo de incorporación a Oportunidades & 9460 & 0.10 & 0.30 & 0 & I \\
\hline 1998 & 9460 & 0.16 & 0.37 & 0 & I \\
\hline $1999-2000$ & 9460 & 0.13 & 0.34 & 0 & I \\
\hline $2001-2003$ & 9460 & 0.22 & 0.41 & 0 & I \\
\hline
\end{tabular}

2004-2007

Del municipio:

\begin{tabular}{llllll}
\hline Densidad de médicos & 9460 & 0.63 & 0.48 & 0 & 1 \\
\hline Densidad de centros de salud & 9460 & 0.40 & 0.49 & 0 & 1 \\
& & & & & \\
\hline
\end{tabular}

medio $(\mathrm{RM}=0.77)$ y alto $(\mathrm{RM}=0.81)$ se relacionan con la falta de realización de pruebas de Papanicolaou al compararlas con mujeres que viven en localidades con un índice de marginación bajo. Al acotar la muestra al grupo de mujeres de más riesgo entre 35 y 49 años se identificaron nexos significativos de la disponibilidad de médicos y unidades de salud, la incorporación y antigüedad de Oportunidades y el aseguramiento con la práctica de cualquiera de los dos exámenes (los resultados no se muestran).

En la tercera columna del cuadro III se muestran los resultados de la realización de alguna mastografía en los 


\section{Cuadro III}

Modelos logit multinivel para el uso de pruebas preVentivas de mastografía y Papanicolaou en los últimos 12 meses en mujeres de 20 a 49 años. México, octubre 2008

Individuales:

Mujer de 20 a 29 años de edad (referencia)

\begin{tabular}{|c|c|c|c|c|}
\hline Mujer de 30 a 39 años de edad & $\begin{array}{c}1.28 I^{*} \\
{[0.099]}\end{array}$ & $\begin{array}{c}1.278^{*} \\
{[0.100]}\end{array}$ & $\begin{array}{c}1.139 \\
{[0.134]}\end{array}$ & $\begin{array}{c}1.185 \\
{[0.138]}\end{array}$ \\
\hline Mujer de 40 a 49 años de edad & $\begin{array}{c}1.494^{*} \\
{[0.134]}\end{array}$ & $\begin{array}{c}1.432^{*} \\
{[0.129]}\end{array}$ & $\begin{array}{c}1.648^{*} \\
{[0.207]}\end{array}$ & $\begin{array}{c}1.736^{*} \\
{[0.216]}\end{array}$ \\
\hline Casada $^{\neq}$ & $\begin{array}{c}1.445^{*} \\
{[0.178]}\end{array}$ & $\begin{array}{c}1.430^{*} \\
{[0.177]}\end{array}$ & $\begin{array}{c}1.134 \\
{[0.197]}\end{array}$ & $\begin{array}{c}1.220 \\
{[0.210]}\end{array}$ \\
\hline Trabaja $^{\infty}$ & $\begin{array}{r}1.192^{\ddagger} \\
{[0.098]}\end{array}$ & $\begin{array}{r}1.176^{\#} \\
{[0.098]}\end{array}$ & $\begin{array}{c}1.065 \\
{[0.112]} \\
0.050\end{array}$ & $\begin{array}{c}1.107 \\
{[0.116]}\end{array}$ \\
\hline Al menos un hijo ${ }^{\circ}$ & $\begin{array}{c}0.889 \\
{[0.076]}\end{array}$ & $\begin{array}{c}0.894 \\
{[0.077]}\end{array}$ & $\begin{array}{c}0.959 \\
{[0.112]}\end{array}$ & $\begin{array}{c}0.944 \\
{[0.109]}\end{array}$ \\
\hline \multicolumn{5}{|l|}{ Sin educación (referencia) } \\
\hline Primaria & $\begin{array}{c}0.650 \\
{[0.735]}\end{array}$ & $\begin{array}{c}0.755 \\
{[0.877]}\end{array}$ & $\begin{array}{c}0.550 \\
{[0.720]}\end{array}$ & $\begin{array}{c}0.494 \\
{[0.646]}\end{array}$ \\
\hline Secundaria & $\begin{array}{c}0.620 \\
{[0.702]}\end{array}$ & $\begin{array}{c}0.716 \\
{[0.834]}\end{array}$ & $\begin{array}{c}0.612 \\
{[0.802]}\end{array}$ & $\begin{array}{c}0.542 \\
{[0.710]}\end{array}$ \\
\hline Superior & $\begin{array}{c}0.672 \\
{[0.768]}\end{array}$ & $\begin{array}{c}0.725 \\
{[0.851]}\end{array}$ & $\begin{array}{c}0.886 \\
{[1.173]}\end{array}$ & $\begin{array}{c}0.800 \\
{[1.058]}\end{array}$ \\
\hline Se realizó alguna mastografia en el último año & & $\begin{array}{c}3.451^{*} \\
{[0.418]}\end{array}$ & & \\
\hline Se realizó algún Papanicolaou en el último año & & & & $\begin{array}{c}3.457^{*} \\
{[0.410]}\end{array}$ \\
\hline \multicolumn{5}{|l|}{ Del hogar: } \\
\hline Incorporado a Oportunidades (autorreporte) & $\begin{array}{c}3.226 * \\
{[0.259]}\end{array}$ & $\begin{array}{c}3.189 * \\
{[0.258]}\end{array}$ & $\begin{array}{c}1.112 \\
{[0.132]}\end{array}$ & $\begin{array}{l}1.423^{*} \\
{[0.166]}\end{array}$ \\
\hline Jefe del hogar o esposa (o) indígena & $\begin{array}{c}0.757^{*} \\
{[0.078]}\end{array}$ & $\begin{array}{c}0.758^{*} \\
{[0.078]}\end{array}$ & $\begin{array}{c}0.671^{*} \\
{[0.094]}\end{array}$ & $\begin{array}{r}0.673^{*} \\
{[0.095]}\end{array}$ \\
\hline \multicolumn{5}{|l|}{ Condición de aseguramiento: } \\
\hline IMSS o ISSSTE & $\begin{array}{r}1.275^{\#} \\
{[0.168]}\end{array}$ & $\begin{array}{c}1.204 \\
{[0.162]}\end{array}$ & $\begin{array}{l}1.937^{*} \\
{[0.310]}\end{array}$ & $\begin{array}{c}1.952 \\
{[0.310]}\end{array}$ \\
\hline IMSS-Oportunidades & $\begin{array}{c}1.197^{*} \\
{[0.113]}\end{array}$ & $\begin{array}{c}1.222^{\ddagger} \\
{[0.117]}\end{array}$ & $\begin{array}{c}0.931 \\
{[0.108]}\end{array}$ & $\begin{array}{c}0.960 \\
{[0.11 \mathrm{II}]}\end{array}$ \\
\hline Seguro Popular & $\begin{array}{c}1.382^{*} \\
{[0.1 / 2]}\end{array}$ & $\begin{array}{c}1.396 \\
{[0.114]}\end{array}$ & $\begin{array}{c}0.901 \\
{[0.094]}\end{array}$ & $\begin{array}{c}0.944 \\
{[0.099]}\end{array}$ \\
\hline Índice de activos (aprox. de nivel socioeconómico) & $\begin{array}{c}1.060 \\
{[0.042]}\end{array}$ & $\begin{array}{c}1.07 I^{\#} \\
{[0.042]}\end{array}$ & $\begin{array}{c}0.940 \\
{[0.049]}\end{array}$ & $\begin{array}{c}0.940 \\
{[0.049]}\end{array}$ \\
\hline
\end{tabular}

Locales:

Grado de marginación local bajo (referencia)

\begin{tabular}{|c|c|c|c|c|}
\hline Grado de marginación local medio & $\begin{array}{r}0.766^{*} \\
{[0.079]}\end{array}$ & $\begin{array}{c}1.324^{\ddagger} \\
{[0.184]}\end{array}$ & $\begin{array}{c}1.083 \\
{[0.157]}\end{array}$ & $\begin{array}{c}0.879 \\
{[0.105]}\end{array}$ \\
\hline Grado de marginación local alto & $\begin{array}{r}0.807^{\#} \\
{[0.097]}\end{array}$ & $\begin{array}{c}1.108 \\
{[0.169]}\end{array}$ & $\begin{array}{c}1.090 \\
{[0.195]}\end{array}$ & $\begin{array}{c}0.830 \\
{[0.120]}\end{array}$ \\
\hline Disponibilidad de un centro de salud & $\begin{array}{r}1.223^{\ddagger} \\
{[0.108]}\end{array}$ & $\begin{array}{r}1.282^{*} \\
{[0.113]}\end{array}$ & $\begin{array}{c}0.970 \\
{[0.100]}\end{array}$ & $\begin{array}{c}0.993 \\
{[0.105]}\end{array}$ \\
\hline \multicolumn{5}{|l|}{ Periodo de incorporación a Oportunidades } \\
\hline 1998 & $\begin{array}{r}1.399 * \\
{[0.176]}\end{array}$ & $\begin{array}{r}1.317^{\ddagger} \\
{[0.167]}\end{array}$ & $\begin{array}{r}1.555^{*} \\
{[0.220]}\end{array}$ & $\begin{array}{r}1.652^{*} \\
{[0.234]}\end{array}$ \\
\hline $1999-2000$ & $\begin{array}{c}1.136 \\
{[0.114]}\end{array}$ & $\begin{array}{c}1.051 \\
{[0.105]}\end{array}$ & $\begin{array}{r}1.502^{*} \\
{[0.187]}\end{array}$ & $\begin{array}{r}1.537^{*} \\
{[0.191]}\end{array}$ \\
\hline $2001-2003$ & $\begin{array}{r}1.244^{\ddagger} \\
{[0.132]}\end{array}$ & $\begin{array}{c}1.165 \\
{[0.123]}\end{array}$ & $\begin{array}{r}1.409 * \\
{[0.183]}\end{array}$ & $\begin{array}{r}1.475^{*} \\
{[0.191]}\end{array}$ \\
\hline \multicolumn{5}{|l|}{ 2004-2007 (referencia) } \\
\hline \multicolumn{5}{|l|}{ Municipales: } \\
\hline Densidad de médicos & $\begin{array}{c}1.184^{\#} \\
{[0.108]}\end{array}$ & $\begin{array}{c}1.132 \\
{[0.103]}\end{array}$ & $\begin{array}{c}1.056 \\
{[0.112]}\end{array}$ & $\begin{array}{c}1.056 \\
{[0.114]}\end{array}$ \\
\hline Densidad de centros de salud & $\begin{array}{c}0.917 \\
{[0.085]}\end{array}$ & $\begin{array}{c}0.88 \mathrm{I} \\
{[0.080]}\end{array}$ & $\begin{array}{l}1.263^{\ddagger} \\
{[0.135]}\end{array}$ & $\begin{array}{c}1.273^{\ddagger} \\
{[0.138]}\end{array}$ \\
\hline \multicolumn{5}{|l|}{ Constante } \\
\hline $\begin{array}{l}\text { Observaciones } \\
\text { Máxima verosimilitud }\end{array}$ & $\begin{array}{c}7428 \\
-3645.9\end{array}$ & $\begin{array}{c}7428 \\
-3583.6\end{array}$ & $\begin{array}{c}7428 \\
-2328.5\end{array}$ & $\begin{array}{c}7428 \\
-2328.5\end{array}$ \\
\hline Rho & $\begin{array}{c}0.112 \\
{[0.015]}\end{array}$ & $\begin{array}{c}0.108 \\
{[0.015]}\end{array}$ & $\begin{array}{c}0.078 \\
{[0.018]}\end{array}$ & $\begin{array}{c}0.078 \\
{[0.018]}\end{array}$ \\
\hline $\begin{array}{ll}\text { Prob }>=\chi^{2} & \chi^{2}(22)\end{array}$ & $\begin{array}{c}204.37 \\
0.000\end{array}$ & $\begin{array}{c}186.5 \\
0.000\end{array}$ & 0.000 & $\begin{array}{l}35.39 \\
0.000\end{array}$ \\
\hline \multicolumn{5}{|c|}{$\begin{array}{l}\text { * } p<0.01,{ }^{\ddagger} p<0.05,+p<0.10 \text {. Errores estándar robustos en corchetes } \\
\text { \& Razón de momios notificada } \\
{ }^{*} \text { Variable igual a uno si la mujer indica que está casada o unida, e igual a cero en caso contrario } \\
{ }^{\infty} \text { Variable igual a uno si la mujer indica que trabajó en la última semana de la fecha en la que se le entrevista, e igual a cero en caso contrario } \\
{ }^{\circ} \text { Variable igual a uno si la mujer indica que al menos tiene un hijo, e igual a cero en caso contrario }\end{array}$} \\
\hline
\end{tabular}


últimos 12 meses. ${ }^{*}$ Las mujeres de 40 a 49 años tienen una vinculación de relevancia estadística $(\mathrm{RM}=1.74)$ con la realización de una mastografía en los últimos 12 meses. ${ }^{\ddagger}$ No se encontraron nexos estadísticamente significativos en el resto de las características individuales de la mujer, pero sí en otras variables como la cobertura de aseguramiento y del programa Oportunidades. Aquellas mujeres aseguradas por el IMSS o el ISSSTE tienen una vinculación significativa con la práctica de mastografía $(R M=1.95)$. Las personas que pertenecen a familias beneficiadas por Oportunidades presentan también una relación notoria con la realización de mastografías $(\mathrm{RM}=1.42)$. Se encontró un mayor nexo entre el periodo de incorporación a Oportunidades y el acceso de los exámenes entre mujeres de localidades con mayor duración en Oportunidades. Al tomar como referencia aquellos que se incorporaron en 2004-2007, la relación de pertenecer a una familia incorporada en 1998 con la realización de mastografías tiene una razón de momios de 1.65, mientras que ésta disminuye en los periodos de 1999-2000 a 1.54 y en 2001-2003 a 1.47.

Las mujeres de condición indígena tienen una vinculación estadísticamente significativa $(\mathrm{RM}=0.67)$ con la falta de realización de mastografía en los últimos

\footnotetext{
* Se estimaron los modelos para Papanicolaou y mastografía en una submuestra de mujeres en el grupo de edad de mayor riesgo de 40 a 49 años, incluida la edad en años en lugar de grupos de edad, con el propósito de analizar si en este grupo se encontraban diferencias estadísticamente significativas. Sin embargo, no se hallaron resultados de relevancia estadística.

* Se efectuó un análisis de sensibilidad tras calcular el modelo sólo con mujeres de 40 a 49 años; empero, no se encontraron diferencias estadísticamente significativas en la realización de mastografías entre mujeres de distintas edades en la submuestra de 40 a 49 años ni tampoco se reconocieron diferencias significativas en el resto de las covariables.
}

12 meses. Por último, la mayor densidad de centros de salud municipales contribuye en grado notorio a la práctica de una mastografía $(\mathrm{RM}=1.27)$.

En el ajuste del modelo de Papanicolaou se incluyó de manera adicional una variable que indica si la mujer se ha sometido a un examen de mastografía. El resultado se muestra en la segunda columna del cuadro III, en la que se observa una relación significativa entre la práctica de la mastografía y el Papanicolaou $(\mathrm{RM}=$ 3.451). Al incluir una variable dicotómica que indica la realización de al menos un examen de Papanicolaou en el modelo multivariado de mastografía se encontró que la práctica de al menos un Papanicolaou se vincula con la realización de mastografías $(\mathrm{RM}=3.46)$.

\section{Resultados del análisis de correlación simple para mujeres de 65 años y mayores}

Con respecto a las mujeres de 65 y más años se identificó que $55 \%$ posee una relación matrimonial o de unión; la mayor parte tiene al menos un hijo $(93 \%)$ y un nivel de educación primaria. Casi 70\% está incorporado al programa de Oportunidades y se ve poco favorecido por algún tipo de aseguramiento: 11\% del IMSS o ISSSTE y $22 \%$ del Seguro Popular. Hasta $73 \%$ vive en alguna localidad con al menos un centro de salud y en zonas de marginación media y baja (datos no mostrados).

Los resultados de las correlaciones simples de la realización del Papanicolaou o la mastografía en los últimos tres años se muestran en el cuadro IV. Las mujeres casadas y con acceso a aseguramiento público (Seguro Popular) tienen una correlación positiva con la práctica del Papanicolaou en los últimos tres años. Dicha correlación fue negativa respecto de la variable de edad. Al igual que las mujeres más jóvenes, las que pertenecen a localidades que se incorporaron más temprano a Oportunidades y localidades que tienen más recursos de

Cuadro IV

Matriz de Correlación entre pruebas PReVentivas en mujeres de 65 y más años e indicadores Selectos. MÉXICO, OCTUBRE 2008

\begin{tabular}{|c|c|c|c|c|c|c|c|c|c|c|c|c|c|c|}
\hline \multirow[b]{2}{*}{ Mastografía } & \multicolumn{2}{|c|}{ Características individuales } & \multicolumn{4}{|c|}{ Año de incorporación a Oportunidades } & \multicolumn{2}{|c|}{ Aseguramiento } & \multicolumn{3}{|c|}{ Nivel de marginación } & \multicolumn{3}{|c|}{ Acceso a servicios de salud } \\
\hline & -0.04 & 0.10 & 0.12 & 0.06 & -0.13 & -0.13 & 0.12 & -0.08 & 0.10 & 0.01 & -0.14 & 0.11 & 0.10 & 0.03 \\
\hline Papanicolaou & -0.08 & 0.28 & 0.11 & 0.04 & -0.01 & -0.17 & 0.11 & 0.02 & 0.15 & -0.05 & -0.15 & 0.14 & 0.25 & 0.15 \\
\hline & 胥 & $\begin{array}{l}\text { 苞 } \\
\text { J }\end{array}$ & 只 & $\begin{array}{l}\text { ठ্ণ } \\
\text { مু } \\
\text { مू }\end{array}$ & 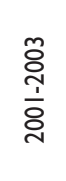 & 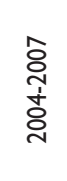 & 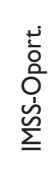 & $\begin{array}{l}\frac{i}{\bar{z}} \\
\frac{\bar{a}}{0} \\
\frac{0}{0} \\
\stackrel{0}{\circ}\end{array}$ & 㖒 & $\frac{\frac{\pi}{\sigma}}{\frac{\pi}{2}}$ & $\frac{\mathbb{H}}{<}$ & 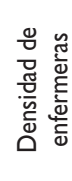 & 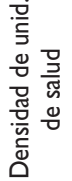 & 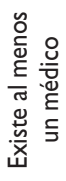 \\
\hline
\end{tabular}


salud poseen una correlación positiva con la realización del Papanicolaou. Por último, las mujeres que viven en localidades con mayores índices de marginación muestran una correlación negativa con la práctica del Papanicolaou.

La edad de las mujeres mayores se correlaciona de forma negativa con la realización de una mastografía en los últimos tres años. Se encontró que variables como la disponibilidad de recursos médicos y unidades de salud tienen una correlación positiva con la práctica de mastografías. Las mujeres que viven en localidades con mayor tiempo en Oportunidades tienen una correlación positiva con la obtención de una mastografía. Finalmente, las mujeres que viven en localidades con un índice de marginación bajo o medio muestran una correlación positiva para la práctica de mastografías, mientras que en aquellas que viven en localidades de alta marginación dicha correlación es negativa.

\section{Discusión y conclusiones}

Las mujeres de mayor edad se sometieron con mayor frecuencia a una mastografía y examen de Papanicolaou en los últimos 12 meses. Sin embargo, la frecuencia con la que se informó la obtención de una mastografía en los últimos 12 meses fue menor al examen de Papanicolaou.

En la realización de una mastografía se encontraron relaciones significativas, sobre todo con variables de acceso a servicios de salud y cobertura de aseguramiento. Las mujeres que viven en municipios con mayor disponibilidad de unidades de salud, cubiertas por seguridad social o Seguro Popular, son más propensas a notificar la práctica de una mastografía en los últimos 12 meses. No se encontraron vinculaciones significativas en variables que indican la condición socioeconómica de la mujer, pero sí la condición indígena como un factor que reduce el informe de la práctica de una mastografía en los últimos 12 meses.

En el caso de la prueba del Papanicolaou se observó que tanto las variables individuales y socioeconómicas como las de disponibilidad de servicios de salud influyen en su realización. La disponibilidad de centros de salud en el plano local y de médicos en el municipal se vincula con la ejecución del examen del Papanicolaou. El mayor grado de marginación en la localidad y la condición indígena son factores de riesgo para la falta de realización del Papanicolaou. En ninguno de los dos casos se identificó un efecto significativo del nivel de educación de la mujer.

Las mujeres beneficiadas por Oportunidades señalan con mayor frecuencia la realización de alguna de las dos pruebas en comparación con aquellas que no se benefi- cian del programa. De forma adicional, la duración en el programa Oportunidades se vincula con la práctica de los dos exámenes, lo que confirma el posible efecto de aprendizaje de las mujeres incorporadas a dicho programa. Sin embargo, cabe resaltar que este efecto puede estar influido por la presencia de otros programas de prevención reproductiva que promueven la realización del Papanicolaou y mastografía en ese periodo. *

Se reconoció que la realización de mastografías es baja y que aspectos relacionados con el acceso a infraestructura de salud y cobertura de aseguramiento influyen de manera positiva en el acceso de mujeres localizadas en zonas rurales. De la misma forma, un indicador de cobertura efectiva es el seguimiento y acceso a servicios entre las pacientes que presentan anormalidades. Al respecto, destaca la elevada proporción de mujeres con resultados anormales que carecieron de seguimiento médico, en particular en personas de los grupos de edad de mayor riesgo ( 40 a 49 años), lo que denota la falta de cobertura efectiva y el alto riesgo de letalidad de esta anomalía en mujeres de áreas rurales.

A pesar de que el informe de la realización de pruebas para la detección de $\mathrm{CaCu}$ es mayor a la encontrada en la mastografía, existen aún diferencias en el acceso a estas pruebas de acuerdo con el nivel socioeconómico del hogar y el acceso a algún tipo de aseguramiento, aspecto que ratifica lo notificado en estudios nacionales. ${ }^{17}$ Esto corrobora la necesidad de fortalecer los programas de prevención de $\mathrm{CaCu}$, en especial en áreas rurales marginadas e indígenas.

La práctica de mastografías y Papanicolaou entre mujeres de 65 años y mayores muestra que las variables indicativas del acceso a servicios de salud se relacionan en grado positivo con la realización de ambas pruebas. En ese grupo, las personas de mayor edad notifican una menor ejecución de mastografías y Papanicolaou. Por la edad, tales personas debían someterse a una mastografía anual dado que se hallan en un grupo de edad de alto riesgo.

Es importante resaltar que no se reconocieron nexos significativos entre las variables de educación de la mujer y el índice de activos del hogar con el acceso a las pruebas del Papanicolaou y la mastografía. Esto podría explicarse por la homogeneidad de la muestra de mujeres y hogares estudiada, lo que tal vez refleje la similitud en el acceso a este tipo de pruebas en los

\footnotetext{
* Al tratar de considerar este posible efecto, los modelos se ajustaron por la presencia de programas de salud, como el Seguro Popular, además de la consideración de los efectos fijos a nivel de localidad.
} 
planos individual y del hogar. En contraste, se encontró una relación significativa con el índice de marginación en la localidad, lo cual puede aludir a la relación entre el acceso a pruebas de mastografía y Papanicolaou y su vinculación con el índice de desarrollo local. ${ }^{*}$

Se mostró la baja frecuencia con la que se lleva a cabo el examen de mastografía en áreas rurales pobres, además de la baja notificación de seguimiento médico entre mujeres de grupos de edad de alto riesgo y con resultados anormales. Es fundamental fortalecer el acceso a la práctica de mastografías entre mujeres de alto riesgo en áreas rurales y garantizar un seguimiento adecuado de los casos anormales para aumentar la detección temprana y con ello reducir el nivel de letalidad del CaMa.

\section{Limitaciones}

El análisis empírico desarrollado en este artículo tuvo algunas limitaciones. De manera inicial, aunque se incluyó a grupos de edad de alto riesgo para padecer $\mathrm{CaCu}$ y CaMa (entre 35 y 49 años), no se contó con información sobre mujeres de 50 a 64 años, un rango de edad de alto riesgo. Asimismo, la información sobre la realización de pruebas de Papanicolaou y mastografía la suministró la mujer, lo que puede llevar, en cierto grado, a introducir cierto sesgo de autorreporte en el análisis empírico.

Dado que las mujeres de 65 y más años recuerdan el acceso a mastografía y Papanicolaou en un periodo de tres años, esto puede inducir algún sesgo de memoria en las respuestas de estas mujeres. Por último, puesto que este artículo se enfocó en analizar el uso de servicios preventivos reproductivos entre mujeres que viven en localidades beneficiadas por Oportunidades, esto pudo sesgar el impacto de las coberturas informadas en ambos exámenes, ya que las mujeres de la muestra estudiada podrían ser más conscientes del uso de servicios reproductivos y las respuestas que deben dar sobre el uso de los servicios preventivos.

\footnotetext{
* El índice de marginación se compone de un grupo de indicadores de desarrollo a nivel localidad más amplio que el índice de bienes construido a nivel hogar. El índice de marginación considera aspectos de educación e infraestructura a nivel localidad (drenaje, piso, electricidad, etc.).
}

\section{Referencias}

I. Peto R, Boreham J, Clarke M, Davies C, Beral V. UK and USA breast cancer deaths down $25 \%$ in year 2000 at ages $20-69$ years. Lancet 2000;355: 1822.

2. Levi F, Bosetti C, Lucchini F, Negri E, La Vecchia C. Monitoring the decrease in breast cancer mortality in Europe. Eur J Can Preven 2005; I4(6): 97-502.

3. Brandan ME,Villaseñor-Navarro Y. Detección del cáncer de mama: estado de la mamografía en México. Cancerología 2006; I (3). [Consultado el 17 de noviembre de 2008]. Disponible en: http://www.incan.org. $\mathrm{mx} /$ revistaincan/index.php? id=| | 722857|3.

4. Secretaría de Salud. Información en Salud. 2008. Disponible en: http:// sinais.salud.gob.mx/descargas/pdf//B_2006.pdf. Consultado: I7/I I/08. 5. Althuis MD, Dozier JM, Anderson WF, Devesa SS, Brinton LA. Global trends in breast cancer incidence and mortality 1973-1997. Int J Epidemiol 2005;34:405-4I2.

6. Secretaría de Salud. Comunicado de Prensa No. 400. México, D.F: 04/Agosto/2005 Disponible en: http://www.salud.gob.mx/ssa_app/noticias/ datos/2005-08-04_I560.html Consultado: I2/0I-2008.

7. World Health Organization. Cervical Cancer Screening in Developing Countries. Report of WHO consultation. 2002. [Consultado el I de noviembre de 2008]. Disponible en: http://www.who.int/cancer/media/en/ cancer_cervical_3732I.pdf.

8. Lazcano-Ponce E,Yunes-Díaz EM. Evolución de las pruebas de tamizaje para la detección oportuna de cáncer. Gamo 2006; (5)4: 91-92.

9. Gutiérrez-Trujillo G, Martínez-Montañez OG, Fernández-Gárate IH, Mejía-Rodríguez I, Reyes-Morales I, Dirección de Prestaciones Médicas. Análisis del descenso de la mortalidad por cáncer cervicouterino en el IMSS, I991-2005. PREVENIMSS 2006; I (44).

10. Hidalgo-Martínez AC. El cáncer cérvico-uterino, su impacto en México y el porqué no funciona el programa nacional de detección oportuna. Rev Biomed 2006; 17:8I-84.

II. Hernández-Peña P, Lazcano-Ponce EC,Alonso-de Ruiz P, Cruz-Valdez A, Meneses-González F, Hernández-Ávila M. Análisis costo beneficio del Programa de Detección Oportuna del Cáncer Cervicouterino. Salud Publica Mex 1997;4(39).

I2. ENCEL. Oportunidades.2007. [Consultado el I5 de octubre de 2008]. Disponible en: http://www.oportunidades.gob.mx.

13. Scoufias E, Davis B, Behrman JR. Evaluación de la selección de hogares beneficiarios en el (Progresa) Programa de Educación, Salud y Alimentación. México: IFPRI, 1999.

14. Rabe-Hesketh S, Skrondal A. Multilevel and longitudinal modeling using Stata. Stata Press 2005.

15. American College of Obstetricians and Gynecologists (ACOG). [Consultado el 25 de octubre de 2008]. Disponible en: http://www.acog. org/publications/committee_opinions/co335.cfm. 2008.

16. Kolenikov S, Angeles $\mathrm{G}$. The use of discrete data in principal component analysis: theory, simulations, and applications to socioeconomic indices. Working Paper of MEASURE/Evaluation. Carolina Population Center, UNC.WP-04-85. 2004.

17. Meneses GF, Lazcano-Ponce EC, Lino-González M, Hernández-Ávila M, Lezana-Fernández MA, Nájera-Aguilar P, et al. Prevalencia de uso de la prueba de Papanicolaou en mujeres de 15 a 49 años en México. Rev Inst Nal Cancerol Mex 1999; 45(I):17-23. 